\title{
Finding Pareto Optimal Insurance Contracts
}

Yuri M. Ermoliev (ermoliev@iiasa.ac.at)

Sjur Didrik Flåm (sjur.flaam@econ.uib.no)

\section{Approved by}

Gordon MacDonald (macdon@iiasa.ac.at)

Director, IIASA

June 2000 


\section{About the Authors and Acknowledgments}

Yuri M. Ermoliev is an Institute Scholar at IIASA, A-2361 Laxenburg, Austria.

Sjur Didrik Flåm is the corresponding author. He is Professor at the Department of Economics, University of Bergen, N-5007 Norway.

We would like to thank Meltzers høyskolefond, Ruhrgas and IIASA for the generous support. 


\begin{abstract}
This note deals with on-line computation or learning of Pareto optimal insurance contracts. We account for the fact that the loss distribution often is unknown, unavailable, or intractable. Alternatively, the contracting parties could be inexperienced. In both cases losses must be simulated or observed, one at a time, these causing iterated revisions of the premium. The mechanical nature of probability calculus thus yields to more tentative procedures, possibly closer to how humans operate or reason in face of risk. Emphasized here is the remarkable simplicity and stability of the resulting procedures. Special attention goes to catastrophic risks and subsidized insurance.
\end{abstract}




\section{Contents}

1 Introduction $\quad 1$

2 The Model 2

3 Avenues to Pareto Optimal Contracts 4

4 The case of Catastrophic Risk $\quad 8$

5 Subsidized Insurance $\quad 9$ 


\title{
Finding Pareto Optimal Insurance Contracts
}

\author{
Yuri M. Ermoliev (ermoliev@iiasa.ac.at) \\ Sjur Didrik Flåm (sjur.flaam@econ.uib.no)
}

\section{Introduction}

It is hard to deal effectively with probabilistic information, particularly with regard to events of sizeable magnitude but infrequent occurrence. Above all, this fact applies to the perception and management of so-called economic risks. The need for such management leads us to consider here how stochastic optimization, and notably Monte Carlo techniques, may assist in designing risk-transfer from exposed, averse agents to specialized bearers. In that consideration we shall focus exclusively on welfare efficient arrangements.

The setting is broadly as follows. A mutual, a syndicate, or a representative agent faces a risk. Specifically, the agent at hand may incur an economic loss of not quite predictable magnitude. Preferring more predictability and less uncertainty he shifts the said risk, in part or full, to an insurer. The latter, who thereby receives a compensating premium, could be the public sector, an insurance company, the capital market, or a composition of those institutions. Such situations, being ubiquitous in reality, are bread and butter for practical insurance - and for its theory. On the normative side of that theory, the Pareto optimal form of risk-shifting has been studied by Arrow [1], Raviv [9] and others. (For a nice exposition see [8].) On the positive side, how those contracts can be computed, or achieved, has received little attention. This motivates us to explore that issue here below.

A premise for our investigation - and a problem as well - is that the risk at hand has unknown or hidden probability distribution. Consequently, our approach can presume no or little knowledge of probabilities and distributions of these. Respecting that shortage, we shall neither evaluate mean values nor require the capacity to do so. Of course, some surrogate and compensating input must then be available. As such we shall repeatedly let realized, representative losses cause some adaptation of the premium. Accordingly, what is set up in the following is an iterative scheme totally driven by upcoming samples or observations. That scheme amounts to adaptive optimization, using Monte Carlo simulation or observed losses. It may be construed as a learning procedure which depicts how inexperienced agents, in terms of premium and coverage, eventually may come up with Pareto optimal insurance. In short, we advocate a step-wise procedure that has two rather attractive features: First, it is remarkably simple in form, concept, and implementation. Second, it effectively and quite naturally splits insurance design in two related parts: an easy ex post specification of recompenses, and a more difficult choice of the appropriate premium 
paid ex ante. If such splitting were not undertaken, then contract design would entail difficult search in a function space.

\section{The Model}

To convey main ideas we confine attention to tractable features, embodying only a small measure of complexity. Thus we consider a stylized setting in which agent 1 holds an economic risk $X$, henceforth called a loss. Formally, this object is a nonnegative random variable defined on some probability space not made explicit here. For simplicity in the argument we posit that $X$ has a probability density $f(x) d x .^{1}$ The function $f(\cdot)$ may very well be unknown. We assume though, that someone has the capacity to simulate (or observe) an infinite sequence $\left\{X_{n}\right\}$ of independent realizations, each distributed as $X$. Members of $\left\{X_{n}\right\}$ will be unveiled and arrive sequentially, one at a time.

At a fixed premium $\pi$ agent 1 buys an insurance policy $I=I(\cdot)$, guaranteeing him recovery (recompense) of the part $I(x) \in[0, \mathcal{I}(x)]$ in case the loss $X=x$ comes up. $\mathcal{I}(x)$ denotes an upper level beyond which additional losses will not be covered. For instance, the specification $\mathcal{I}(x):=\min \{x, L\}$ reflects presence of a cap-loss $L$. If no such cap exists, then take $L=+\infty$ and $\mathcal{I}(x)=x$.

We shall focus on the treaty $(\pi, I)$ but take no organized insurance market as given; the pair $(\pi, I)$ could rather be seen as a customized contract. Agent 1 has wealth $W_{1}$ and von Neumann-Morgenstern utility $U_{1}[\cdot]$ with $U_{1}^{\prime}>0, U_{1}^{\prime \prime}<0$. So, if insured in such terms, he envisages expected utility

$$
\bar{U}_{1}(\pi, I):=\int U_{1}\left[W_{1}-\pi-x+I(x)\right] f(x) d x .
$$

Insurance will limit his losses but also support his economical activities in ways not modelled here. Suppose henceforth that the pair $(\pi, I)$ makes insurance worthwhile. That is, suppose $\bar{U}_{1}(\pi, I) \geq \bar{U}_{1}(0,0)=\int U_{1}\left[W_{1}-x\right] f(x) d x$. This inequality must hold to incite demand for the associated contract. On the supply side resides agent 2 , also called the insurer. He issues and underwrites the policy $I$. Enjoying wealth (or reserves) $W_{2}$ and von Neumann-Morgenstern utility $U_{2}[\cdot]$ he obtains in expectation

$$
\bar{U}_{2}(\pi, I):=\int U_{2}\left[W_{2}+\pi-I(x)-c(I(x))\right] f(x) d x
$$

where $c(\cdot)$ accounts for his administrative cost. We posit that $U_{2}$ and $c$ be twice continuously differentiable with

$$
U_{2}^{\prime}>0, U_{2}^{\prime \prime} \leq 0, \text { and } c^{\prime}>-1, c^{\prime \prime} \geq 0 .
$$

Given coexistence of these two agents our errand is to the compute, or constructively approach, Pareto optimal insurance contracts. Motivation for this inquiry stems mainly from two sources: First, it is interesting in itself to exhibit what policies

\footnotetext{
${ }^{1}$ This assumption is not necessary and will later be relaxed.
} 
will maximize total welfare under diverse circumstances. Second, we note that novel insurance instruments are now traded in fairly competitive, large, and widespread financial markets. The first fundamental welfare theorem indicates that equilibrium, under such price-taking conditions, might come fairly close to Pareto optimum. Anyway, the problem of finding a Pareto optimal pair $(\pi, I)$ can now be stated as follows:

$$
\left.\begin{array}{ll}
\operatorname{maximize} & \bar{U}_{1}(\pi, I) \\
\text { subject to } & \bar{U}_{2}(\pi, I) \geq u_{2}
\end{array}\right\}
$$

where $u_{2} \geq \bar{U}_{2}(0,0)=U_{2}\left[W_{2}\right]$ is a specified constant, guaranteeing the insurer a threshold welfare. Alternatively, one may fix weights $\alpha_{1} \in(0,1), \alpha_{2}:=1-\alpha_{1}$, and consider the problem

$$
\text { maximize } \quad \alpha_{1} \bar{U}_{1}(\pi, I)+\alpha_{2} \bar{U}_{2}(\pi, I)
$$

In fact, we shall mostly deal with formulation (4). The decision variables are, of course, the premium $\pi$ and the recompense function $x \mapsto I(x) \in[0, \mathcal{I}(x)]$. Before addressing (4) we make some

\section{Remarks :}

- (On the objective of the insured) As said, $U_{1}$ could represent the aggregate preference of a finite syndicate or mutual $M$; see [15]. For example, $U_{1}$ might be synthesized as follows: Suppose member $m \in M$ has wealth $w_{m}$, increasing smooth strictly concave utility $u_{m}[\cdot]$, and risk $X_{m}$. Then, let $W_{1}:=\sum_{m \in M} w_{m}$, $X:=\sum_{m \in M} X_{m}$, and

$$
U_{1}\left[W_{1}+y\right]:=\sup \left\{\sum_{m \in M} u_{m}\left(w_{m}+y_{m}\right): \sum_{m \in M} y_{m}=y\right\} .
$$

This construction preserves continuity (in fact, differentiability) and concavity.

- (On the objective of the insurer) The first part of assumption (2), concerning the nature of $U_{2}$, is far from innocuous. Specifically, the insurer is apt to worry about insolvency (and a fortiori about potential ruin). If so, a chance constraint

$$
\operatorname{Prob}\left\{W_{2}+\pi-I(x)-c(I(x)) \leq 0\right\} \leq r
$$

might be attached to (1), featuring some reasonably small ruin probability $r \in(0,1)$; see [14]. In other words, the insurer's essential objective equals (1) when (5) holds, $-\infty$ otherwise. Such "death penalty" on his part is, of course, unrealistic. Moreover, it may render problems (3) and (4) discontinuous or entail loss of concavity. So, for greater realism and easier computation, the softer penalty and function

$$
\bar{U}_{2}(\pi, I)+\mu E \min \left\{0, W_{2}+\pi-I(x)-c(I(x))\right\}
$$

comes conveniently, incorporating a parameter $\mu>0$. Continuity and concavity will thus be maintained, and for large enough $\mu$ satisfaction of (5) obtains to desirable degree; see [5], [6]. Since $x \mapsto I(x)+c(I(x))$ increases, insolvency occurs when $x$ exceeds some implicit level $\hat{x}=\hat{x}\left(W_{2}, \pi, I(\cdot), c(\cdot)\right)$. So, (5) and $\operatorname{Prob}\{x \geq \hat{x}\} \leq r$ are equivalent. In other words: (5) imposes a chance constrained version of $I(x) \leq I(\hat{x})$. 
- (On uncertainty) Uncertainty is here objective and independent of the agents. We have thus avoided all problems related to subjective probabilities and information asymmetries. Admittedly, this situation seems exceptional. Often the insured holds lower estimates of expected loss than does the insurer. Additional complexity stems from the fact that risks are not entirely exogenous to economic behavior: Their severity depends on mitigation measures not considered here. Also, since uncertainty usually concerns commodity bundles or money payments over various locations and future dates, a multi-variate probability distribution would suit better. However, given existence of markets - and also our focus on economic losses - it appears reasonable to aggregate diverse items and risks by their monetary values.

- (On insurance problems) Since our emphasis is on welfare, we disregard adverse effects of moral hazard, selection, or fraudulent behavior. In particular, we do not discuss whether ex post relief discourages mitigation. Non-insurable risks are also ignored [2]. These may be associated with the considered one, and they would call for multi-variate statistics. We tacitly presume that the costs of information acquisition, contract writing, condition monitoring, and policy enforcement do not preclude purchase of insurance. Finally, the fact that many insurance markets often are politicized, and involve redistribution of wealth, is not accounted for - except in the final section.

\section{Avenues to Pareto Optimal Contracts}

We shall utilize a simple observation, namely: For given premium $\pi$ and loss $x$ the optimal recompense $I(x)$ is easily found. To see this recall that (4) embodies the integrand

$$
\alpha_{1} U_{1}\left[W_{1}-\pi-x+I\right]+\alpha_{2} U_{2}\left[W_{2}+\pi-I-c(I)\right] .
$$

So, given premium $\pi$ and loss $x$, expression (6) should be maximal with respect to $I=I(x) \in[0, \mathcal{I}(x)]$. This elementary fact tells that the real challenge, be it in terms of computation or learning, comes with fixing the premium. We shall address that challenge shortly. First we review though, some well known results concerning the choice of $I$. Since (6) is strictly concave in $I$, the maximizing coverage will be unique. Given smoothness of all intervening functions, the standard first-order optimality conditions becomes both necessary and sufficient. Arrow [1] and Raviv [9] have thus obtained, in one form or another, the following two propositions.

Proposition 1 (Optimal coverage) Let here $x \geq 0$ and $\pi$ be fixed. Suppose $f(x)>$ 0 .

(i) If the unique solution I of

$$
\alpha_{1} U_{1}^{\prime}\left[W_{1}-\pi-x+I\right]=\alpha_{2} U_{2}^{\prime}\left[W_{2}+\pi-I-c(I)\right]\left\{1+c^{\prime}(I)\right\}
$$

belongs to the feasible domain $[0, \mathcal{I}(x)]$, then that $I=I(x)$ is the optimal coverage in case of loss $x$.

(ii) If, on the other hand

$$
\alpha_{1} U_{1}^{\prime}\left[W_{1}-\pi-x\right]<\alpha_{2} U_{2}^{\prime}\left[W_{2}+\pi-c(0)\right]\left\{1+c^{\prime}(0)\right\}
$$


then minimal coverage $I(x)=0$ becomes optimal. More generally, suppose

$$
\alpha_{1} U_{1}^{\prime}\left[W_{1}-\pi\right]<\alpha_{2} U_{2}^{\prime}\left[W_{2}+\pi-c(0)\right]\left\{1+c^{\prime}(0)\right\} .
$$

Then there is a stop-loss level $l$, uniquely determined by

$$
\alpha_{1} U_{1}^{\prime}\left[W_{1}-\pi-l\right]=\alpha_{2} U_{2}^{\prime}\left[W_{2}+\pi-c(0)\right]\left\{1+c^{\prime}(0)\right\},
$$

such that $I(x)=0$ iff $x \leq l$.

(iii) Finally, if

$$
\alpha_{1} U_{1}^{\prime}\left[W_{1}-\pi\right]>\alpha_{2} U_{2}^{\prime}\left[W_{2}+\pi-x-c(x)\right]\left\{1+c^{\prime}(x)\right\}
$$

then maximal coverage $I(x)=\mathcal{I}(x)$ becomes optimal.

Optimal coverage was first studied by Mossin [10] and Smith [13]. Studies of the optimal deductible $l$ include [3] and [11]. Evidently, since $U_{1}^{\prime}$ decreases, and since

$$
U_{2}^{\prime}\left[W_{2}+\pi-x-c(x)\right]\left\{1+c^{\prime}(x)\right\} \geq U_{2}^{\prime}\left[W_{2}+\pi-c(0)\right]\left\{1+c^{\prime}(0)\right\}
$$

it cannot happen that (ii) holds for some loss $x$ while (iii) comes into vigor for others. In other words: at most one, and always the same, of the scenarios (ii) and (iii) will be realized if any.

It is sometimes instructive reconsider the form of $I(\cdot)$ via problem (3). Note that the integral constraint $\bar{U}_{2}(\pi, I) \geq u_{2}$ will bind. Therefore, while having optimal control in mind, we introduce the state variable

$$
s(x):=\int_{0}^{x} U_{2}\left[W_{2}+\pi-I(\cdot)-c(I(\cdot))\right] f(\cdot) .
$$

Note that

$$
s(0)=0 \text { and } s(1)=u_{2} .
$$

Thus, the active constraint $\bar{U}_{2}(\pi, I)=u_{2}$ assumes the equivalent differential form

$$
s^{\prime}(x)=U_{2}\left[W_{2}+\pi-I(x)-c(I(x))\right] f(x)
$$

subject to boundary conditions (7). Using $I$ as decision variable, problem (3), when stated in control form, has Hamiltonian

$$
H(s, I, \lambda):=\left\{U_{1}\left[W_{1}-\pi-x+I\right]+\lambda U_{2}\left[W_{2}+\pi-I-c(I)\right]\right\} f(x) .
$$

Since $H$ does not depend on $s$, the adjoint system $\lambda^{\prime}(x)=-\frac{\partial}{\partial s} H=0$, tells that $\lambda$ must be constant. Let

$$
\begin{aligned}
& r_{1}:=-U_{1}^{\prime \prime}\left[W_{1}-\pi-x+I\right] / U_{1}^{\prime}\left[W_{1}-\pi-x+I\right] \text { and } \\
& r_{2}:=-U_{2}^{\prime \prime}\left[W_{2}+\pi-I-c(I)\right] / U_{2}^{\prime}\left[W_{2}+\pi-I-c(I)\right]
\end{aligned}
$$

denote Arrow-Pratt measures of absolute risk aversion [12]. Pointwise maximization of (8) with respect to $I$ now yields: 
Proposition 2 (Incremental coverage) If the unique solution I to

$$
U_{1}^{\prime}\left[W_{1}-\pi-x+I\right]=\lambda U_{2}^{\prime}\left[W_{2}+\pi-I-c(I)\right]\left\{1+c^{\prime}(I)\right\}
$$

satisfies $I \in[0, \mathcal{I}(x)]$, then that $I$ is the optimal coverage (or recovery rate) of damage $x$. Consequently, the differential equation

$$
I^{\prime}(x)=\frac{r_{1}}{r_{1}+r_{2}\left\{1+c^{\prime}\right\}+\frac{c^{\prime \prime}}{\left(1+c^{\prime}\right)}}
$$

holds in open $x$-regions where (9) is satisfied for some $I(x) \in[0, \mathcal{I}(x)]$. In particular, when the insurer is risk-neutral, we may set $U_{2}^{\prime}=1$ so that (9) and (10) simplify, respectively, to

$$
U_{1}^{\prime}\left[W_{1}-\pi-x+I\right]=\lambda\left\{1+c^{\prime}(I)\right\} \quad \text { and } \quad I^{\prime}(x)=\frac{\left(1+c^{\prime}\right) r_{1}}{\left(1+c^{\prime}\right) r_{1}+c^{\prime \prime}}
$$

If moreover, $c^{\prime}(I)$ equals a constant called load, then

$$
U_{1}^{\prime}\left[W_{1}-\pi-x+I\right]=\lambda\{1+\text { load }\} \quad \text { and } \quad I^{\prime}(x)=1 .
$$

Equation (10) may help in extrapolating $I(\cdot)$ over intervals of substantial size. Anyway, with $I(\cdot)$ holding so much structure one might be tempted to parametrize this item and thereafter proceed to optimize in parameter space. Such temptation should be resisted: It may force $I(\cdot)$ into a straight-jacket, bring no simplification, and overthrow useful concavity properties. Attention and effort should rather concentrate on the premium. The reason is simple: For given $\pi$ the optimal recompense scheme $x \mapsto I(x) \in[0, \mathcal{I}(x)]$ is determined ex post, term by term, for each $x$, as the unique maximand of (6). Moreover, as explained in Proposition 1 and elaborated in Proposition 2, that maximization is easy to execute. So, exploiting this feature let

$$
U(\pi, x):=\max _{0 \leq I \leq \mathcal{I}(x)}\left\{\alpha_{1} U_{1}\left[W_{1}-\pi-x+I\right]+\alpha_{2} U_{2}\left[W_{2}+\pi-I-c(I)\right]\right\}
$$

denote the ex post optimal value. ${ }^{2}$ Program (11) has been stated rather abstractly. In practice it involves concrete and familiar objects such as deductibles and caps. Now the Pareto optimal premium $\pi$ is found as the optimal solution of the program:

$$
\operatorname{maximize} U(\pi):=\int U(\pi, x) f(x) d x
$$

The last observation leads us to record a few crucial properties of the objective in (12). Evidently, for given $x$ integrand (6) is jointly concave in $(\pi, I)$. Consequently, after maximizing with respect to $I$, the resulting optimal value $U(\pi, x)$ remains concave in $\pi$. Differentiability of that value derives directly from Danskin's envelope theorem. So, we get a technically motivated

\footnotetext{
${ }^{2} U_{1}$ and $U_{2}$ could here contain penalty terms stemming from concerns with survival versus ruin. Note that uncertainty does not intervene directly in this part of the problem. Indirectly though, it works via $\pi$.
} 
Proposition 3 (Concavity and differentiability) The reduced function $U(\pi, x)$, as defined in (11), is concave and differentiable in $\pi$ with

$$
\frac{\partial}{\partial \pi} U(\pi, x):=-\alpha_{1} U_{1}^{\prime}\left[W_{1}-\pi-x+I(x)\right]+\alpha_{2} U_{2}^{\prime}\left[W_{2}+\pi-I(x)-c(I(x))\right]
$$

Here $I(x)$ denotes the unique optimal coverage in situation $\pi, x$. It follows that (12) has a concave differentiable objective with

$$
U^{\prime}(\pi):=\int \frac{\partial}{\partial \pi} U(\pi, x) f(x) d x
$$

Another technicality deserves mention here. Namely, even when $U(\pi, x)$ is nonsmooth in $\pi$, integration with respect to a smooth density $f$ restores classical differentiablity - and ensures validity of (14). The objective in (12), its amenable properties notwithstanding, still poses two problems. First, and most severely, we need not know the density $f$. Indeed, the contracting parties may hold little experience - or so far not have identified $f$ with reasonable confidence. Second, absent an analytical form of $f$, exact integration may be out of question. To circumvent these problems we resort to

\section{An iterative procedure, searching for a Pareto efficient premium}

Start at any reasonable guess $\pi_{0}$ - determined maybe by preceding experience.

Update the premium sequentially, at stages $n=0,1, \ldots$, by the rule

$$
\pi_{n+1}:=\pi_{n}+\rho_{n} \frac{\partial}{\partial \pi} U\left(\pi_{n}, X_{n}\right)
$$

The numbers $\rho_{n}>0$ which figure in (15), are predetermined step-sizes, satisfying $\sum \rho_{n}=+\infty$ and $\sum \rho_{n}^{2}<+\infty$. For example, the specification $\rho_{n}=\frac{1}{n+1}$ would suit. In (15) $X_{n}$ is a stage- $n$ independent observation of $X$, possibly furnished by a simulation device. The following result is now a direct consequence of stochastic programming theory [4]:

Proposition 4 (Global convergence) Suppose the sequence $\left\{\pi_{n}\right\}$ generated by (15) remains bounded. Then it converges almost surely to a Pareto optimal premium.

Some remarks fit here:

- Having eventually reached the limiting $\pi$, the associated recompense scheme $x \mapsto I(x)$ is easily constructed. It will then resurge in familiar form.

- The existence of a density $f$ is not needed. For example, if the loss $X$ is distributed over only a finite set of possible outcomes $x$ with associated but unknown probabilities $p(x)$, then the above arguments (except those related to Proposition 2 ) all hold verbatim, using the "integral" $\sum_{x} \cdot p(x)$ instead of $\int \cdot f(x) d x$. Mixed distributions are also important. Section 4 provides illustration.

- State-dependent utility functions can easily be accommodated. Such dependence creates no novel problem for the main argument. Smoothness (i.e., differentiability) of the functions $U_{1}, U_{2}$, and $c$ can be dispensed with. Absent such properties, 
algorithm (15) would still work with generalized derivatives $\frac{\partial}{\partial \pi} U\left(\pi_{n}, X_{n}\right)$ taken in the sense of convex analysis.

- The strict concavity of $U_{1}$ is most natural and convenient. We can do without though, again at the possible expense of loosing classical differentiability.

- Typically, there will be a non-degenerate interval closed $\Pi$ to which the premium $\pi$ a priori must belong. If so, then (15) could, with no loss of convergence properties, safely and better be cast in the form

$$
\pi_{n+1}:=P\left[\pi_{n}+\rho_{n} \frac{\partial}{\partial \pi} U\left(\pi_{n}, X_{n}\right)\right] .
$$

Here $P[r]$ denotes the projection of $r \in \mathbb{R}$ onto the closest point in $\Pi$.

\section{The case of Catastrophic Risk}

We consider here the special instance when agent 1 owns a sizeable capital stock $K>0$ exposed to catastrophic risk. That agent could be a captive or syndicate created by similar or diverse businesses with the shared objective of controlling their insurance cost. Alternatively, the agent in question could be a region or society. Then, for interpretation, one may construe $K$ as measuring the infrastructure threatened by natural catastrophes. That infrastructure serves productive purposes or it provides access to public goods. Thus, if not reduced or destroyed, it generates utility $U_{1}\left[W_{1}+K\right]$ to its risk-averse owner.

A catastrophe will occur with small but presumably known probability $p \in(0,1)$. When it occurs, the fraction $x \in[0,1]$ of $K$ will be wiped out - and rendered worthless - with probability density $f(x)>0$. At a fixed premium $\pi$ per unit of risk-exposed capital its owner may buy an insurance policy $I=I(\cdot)$, guaranteeing him recovery of the fraction $I(x) \in[0, \mathcal{I}(x)] \subseteq[0, x]$ after a catastrophe of size $x .^{3}$ If insured, the capital owner envisages expected utility

$\bar{U}_{1}(\pi, I):=(1-p) U_{1}\left[W_{1}+K(1-\pi)\right]+p \int_{0}^{1} U_{1}\left[W_{1}+K(1-x-\pi+I(x))\right] f(x) d x$.

The insurer obtains in expectation

$\bar{U}_{2}(\pi, I):=(1-p) U_{2}\left[W_{2}+K\{\pi-c(0)\}\right]+p \int_{0}^{1} U_{2}\left[W_{2}-K\{I(x)+c(I(x))-\pi\}\right] f(x) d x$.

For given premium $\pi$ and catastrophe $x$ let

$\hat{U}(\pi, x):=\max _{0 \leq I \leq \mathcal{I}(x)}\left\{\alpha_{1} U_{1}\left[W_{1}+K(1-x-\pi+I)\right]+\alpha_{2} U_{2}\left[W_{2}-K\{I+c(I)-\pi\}\right]\right\}$

denote the post-event Pareto optimal welfare. Insert for that scenario the maximizing recompense $I=I(x)$ to get marginal welfare

$\frac{\partial}{\partial \pi} \hat{U}(\pi, x)=-\alpha_{1} U_{1}^{\prime}\left[W_{1}+K(1-x-\pi+I(x))\right]+\alpha_{2} U_{2}^{\prime}\left[W_{2}-K\{I(x)+c(I(x))-\pi\}\right]$

\footnotetext{
${ }^{3}$ Concerning the financing of catastrophe risk see [7].
} 
per unit of $K$. In terms of the latter entity computation (or learning driven by a flow of data) could proceed as follows:

At stage $n=0,1, \ldots$, given a current premium $\pi_{n}$, sample a new realization $X_{n}$ of $X$. Then update the premium as follows:

$$
\pi_{n+1}:=\pi_{n}+\rho_{n} K\left\langle\begin{array}{c}
(1-p)\left\{-\alpha_{1} U_{1}^{\prime}\left[W_{1}+K\left(1-\pi_{n}\right)\right]+\alpha_{2} U_{2}^{\prime}\left[W_{2}+K\left\{\pi_{n}-c(0)\right\}\right]\right\} \\
+p \frac{\partial}{\partial \pi} \hat{U}\left(\pi_{n}, X_{n}\right)
\end{array}\right\rangle
$$

The sequence so constructed converges to a Pareto optimal premium.

\section{$5 \quad$ Subsidized Insurance}

Insurance often incorporates transfers between groups. This motivates us to conclude by briefly considering problem format (3) with the special interpretation that $-u_{2}$ equals an average subsidy from group 2 , say society at large, to group 1 , for example sick people. Assign a multiplier $\lambda \geq 0$ to the constraint in (3). The resulting Lagrangian then incorporates the integrand

$$
U_{1}\left[W_{1}-\pi-x+I\right]+\lambda\left\{U_{2}\left[W_{2}+\pi-I-c(I)\right]-u_{2}\right\},
$$

much like (6). Given $\pi, x$, and $\lambda$, maximize (16) with respect to $I \in[0, \mathcal{I}(x)]$. The best choice $I(x)$ yields optimal value

$$
U(\pi, x, \lambda):=\max _{0 \leq I \leq \mathcal{I}(x)}\left\langle U_{1}\left[W_{1}-\pi-x+I\right]+\lambda\left\{U_{2}\left[W_{2}+\pi-I-c(I)\right]-u_{2}\right\}\right\rangle
$$

and associated partial derivatives

$$
\begin{aligned}
& \frac{\partial}{\partial \pi} U(\pi, x, \lambda)=-U_{1}^{\prime}\left[W_{1}-\pi-x+I(x)\right]+\lambda U_{2}^{\prime}\left[W_{2}+\pi-I(x)-c(I(x))\right] \\
& \frac{\partial}{\partial \lambda} U(\pi, x, \lambda)=U_{2}\left[W_{2}+\pi-I(x)-c(I(x))\right]-u_{2}
\end{aligned}
$$

We seek a max-min saddle point $(\pi, \lambda)$ of the concave-convex Lagrangian $U(\pi, \lambda):=$ $\int U(\pi, x, \lambda) f(x) d x$. Using the preceding step sizes $\rho_{n}$ and realizations $X_{n}$ such a point might be found as the limit of the following iterations: Start at any reasonably guessed nonnegative pair $\left(\pi_{0}, \lambda_{0}\right)$. Update the premium and the multiplier sequentially, at stages $n=0,1, \ldots$, by the rule

$$
\begin{aligned}
\pi_{n+1} & :=\pi_{n}+\rho_{n} \frac{\partial}{\partial \pi} U\left(\pi_{n}, X_{n}, \lambda_{n}\right), \\
\lambda_{n+1} & :=\max \left\{0, \lambda_{n}-\rho_{n} \frac{\partial}{\partial \lambda} U\left(\pi_{n}, X_{n}, \lambda_{n}\right)\right\} .
\end{aligned}
$$

Then the averaged sequence $\left\{\rho_{0}\left(\pi_{0}, \lambda_{0}\right)+\cdots+\rho_{n}\left(\pi_{n}, \lambda_{n}\right)\right\} /\left\{\rho_{0}+\cdots+\rho_{n}\right\}$ will cluster to a saddle point.

\section{References}

[1] K. Arrow, Uncertainty and the welfare economics of medical care, The American Economic Review 53, 941-969 (1963). 
[2] N. A. Doherty and H. Schlesinger, Optimal insurance in incomplete markets, Journal of Political Economy 91, 6, 1045-1054 (1983).

[3] J. Drèze, Inferring risk tolerance from deductibles in insurance contracts, Cahiers de Geneve 20, 48-52 (1981).

[4] Y. M. Ermoliv and R. J.-B. Wets (Eds.) Numerical Techniques for Stochastic Optimization, Springer-Verlag, Berlin (1988).

[5] Y. M. Ermoliev and V. I. Norkin, On nonsmooth and discontinuous problems of stochastic systems optimization, European Journal of Operations Research 101, 230244 (1997).

[6] Y. M. Ermoliev, T. Y. Ermolieva, G. J. MacDonald, and V. I. Norkin, Insurability of catastrophic risks: The stochastic opimization model, Optimization 47, 251-265 (2000).

[7] K. A. Froot (ed.), The Financing of Catastrophe Risk, The University of Chicago Press, Chicago (1999).

[8] D. Henriet and J.-C. Rochet, Microeconomie de l'assurance, Economica, Paris (1991).

[9] A. Raviv, The design of an optimal insurance policy, The American Economic Review 69, 1, 84-96 (1979).

[10] J. Mossin, Aspects of rational insurance purchasing, Journal of Political Economy $76,4,553-568$ (1968).

[11] B. Pashigian, L. Schkade, and G. Menefee, The selection of an optimal deductible for a given insurance policy, Journal of Business 39, 35-44 (1966).

[12] J. Pratt, Risk aversion in the small and the large, Econometrica 32, 122- 136 (1964).

[13] V. L. Smith, Optimal insurance coverage, Journal of Political Economy 76, 68-77 (1968).

[14] J. M. Stone, A theory of capacity and the insurance of catastrophic risks, Journal of Risk and Insurance 40, 2, 231-244 (1973).

[15] R. Wilson, The theory of syndicates, Econometrica 36, 1, 119-132 (1968). 